\title{
Correction: Upregulation of long non-coding RNA PIncRNA-1 promotes proliferation and induces epithelial-mesenchymal transition in prostate cancer
}

\author{
Yang Jin ${ }^{1,3}$, Zilian Cui ${ }^{2,3}$, Xudong $\mathrm{Li}^{4}$, Xunbo Jin $^{3}$ and Jian Peng ${ }^{1}$ \\ ${ }^{1}$ Department of Hepatobiliary Surgery, Xiangya Hospital, Central South University, Changsha, Hunan, China \\ ${ }^{2}$ Shandong University School of Medicine, Jinan, Shandong, China \\ ${ }^{3}$ Minimally Invasive Urology Center, Shandong Provincial Hospital affiliated to Shandong University, Jinan, Shandong, China \\ ${ }^{4}$ Department of Urology, Binzhou People's Hospital, Binzhou, Shandong, China
}

Published: August 27, 2019

Copyright: Jin et al. This is an open-access article distributed under the terms of the Creative Commons Attribution License 3.0 (CC BY 3.0), which permits unrestricted use, distribution, and reproduction in any medium, provided the original author and source are credited.

These articles have been corrected: The Figure $2 \mathrm{G}$ in 'Upregulation of long non-coding RNA PlncRNA-1 promotes proliferation and induces epithelial-mesenchymal transition in prostate cancer,' (https://doi.org/10.18632/oncotarget.15318), is a duplication of Figure 3F in the published article 'PlncRNA-1 induces apoptosis through the Her-2 pathway in prostate cancer cells,' (https://doi.org/10.4103/1008-682X.178849). The proper Figure 2G is shown below. The authors declare that these corrections do not change the results or conclusions of this paper.

A

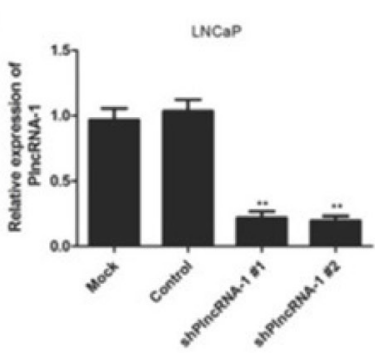

E

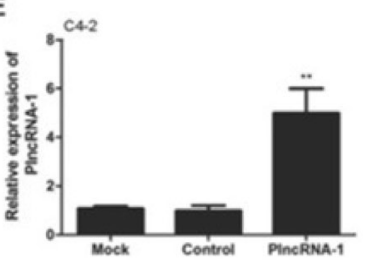

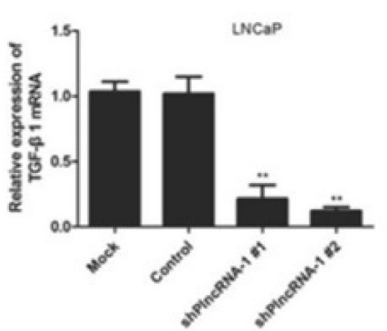

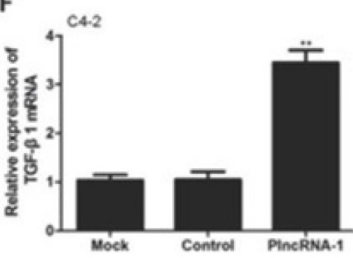

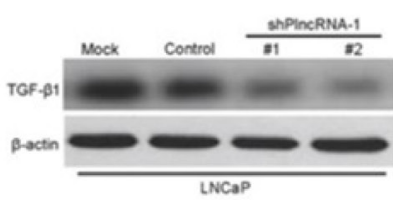

D

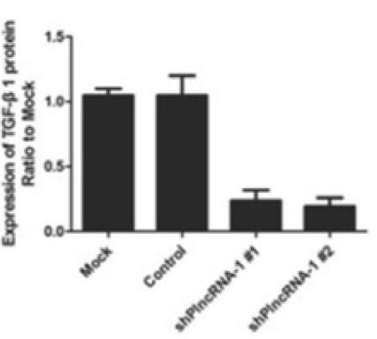

G

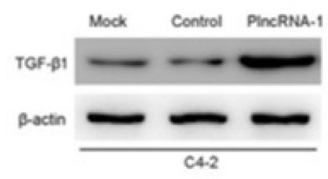

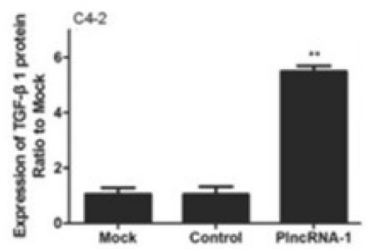

Figure 2: TGF- $\beta 1$ expression in LNCaP and C4-2 cells after silencing and overexpression of PlncRNA-1. A., B. Knockdown of PlncRNA-1 decreased TGF- $\beta 1$ expression, as assessed by qPCR in LNCaP cells. C., D. Knockdown of PlncRNA-1 decreased TGF- $\beta 1$ expression, as assessed by Western blot in LNCaP cells. E., F. Overexpression of PlncRNA-1 upregulated TGF- $\beta 1$, as assessed by qPCR in C4-2 cells. G., H. Overexpression of PlncRNA-1 upregulated TGF- $\beta 1$ expression, as assessed by Western blot in C4-2 cells.

Original article: Oncotarget. 2017; 8:26090-26099. https://doi.org/10.18632/oncotarget.15318 